\title{
Burden movement in confined drift wall blasting tests studied at the LKAB Kiruna SLC mine
}

\author{
M. Wimmer \& A. Nordqvist \\ LKAB, Kiruna, Sweden \\ F. Ouchterlony \& U. Nyberg \\ Swebrec at Luleå University of Technology, Luleå, Sweden
}

J.K. Furtney

Itasca Consulting Group, Minneapolis, USA

\begin{abstract}
Blasting in SLC (sublevel caving) takes place under varying confinement. Blasted material swells while the caved material compacts, and also, to a lesser extent, fills parts of the void volume of the production drift. Several analytical and empirical models have been developed in the past. However, understanding of the interaction of confined blasting conditions, SLC blast design and rock mass characteristics on rock breaking performance is rudimentary. Instrumentation of the blasted burden with various sensors and study of the dynamics of burden movement against confinement is therefore important. Such measurements are very scarce and thus different systems have been tested both in laboratory and in the field. Main focus was thereby the development of measuring equipment that could be scaled up to full-scale SLC blasting and installed behind the rings. In the field tests, blastholes were drilled in a pillar, parallel to a cross cut drift in the LKAB Kiruna SLC mine. The burden constraint was achieved either by filling the drift of $7.0 \times 5.2 \mathrm{~m}$ (width $\times$ height) with rock masses or reinforcing the drift wall. The tests were instrumented with different redundant sensors, which were installed in holes drilled from a parallel cross cut. The most promising one was a concept, which combines the initial movement recorded by an accelerometer designed to minimize zero-shift with the final displacement recorded by a fibre photoelectric sensor ('fibre-optic zebra gauge'). For the filled drift wall blasting test the maximum velocity varied between $16-32 \mathrm{~m} / \mathrm{s}$ with a compaction in the range of $4-5 \%$. A gap was verified to exist between the intact pillar and the blasted material. By comparison, the velocity for an unconfined situation yielded a considerably increased velocity $(42-47 \mathrm{~m} / \mathrm{s})$. In addition, 'Blo-Up', a component of the Hybrid Stress Blast Model (HSBM), was used to model the blasting results. The objective was to show that reasonable predictions of fragmentation and burden movement under confined conditions can be made. An unconfined reference experiment was used to calibrate Blo-Up and forward predictions of the confined case were made. In both cases, Blo-Up accurately reproduced the burden velocity and displacement observed in the experiments. The suggested measurement concept could be used for further measurements of confined burden movement in filled drift wall blasting tests and under controlled situations. As mentioned the final objective is instrumentation of the burden in SLC production rings.
\end{abstract}

\section{INTRODUCTION}

Blasting in Sublevel Caving (SLC) has been identified throughout the literature to have a significant impact upon material flow characteristics and therefore on the overall performance of the SLC method. Brunton (2009) gives an excellent literature review. Blasting in SLC takes place in a situation with varying confinement. Blasted material swells while the caved material compacts, and also, to a lesser extent, fills parts of the void volume of the production drift. Its dynamic behaviour is dependent upon several parameters. Given factors are on the one hand material properties of both the blasted and compressible material. Controllable factors on the other side include the ring layout and blast design.

A number of analytical models and physical experiments were deployed in the past to either quantify the fragmentation or understand the dynamic process of blasting into a compressible material. With a limited number of small-scale tests primarily the final swell and compaction were investigated (Belen'kii 1969, Rustan 1970, Cullum 1974, Volchenko 1977, Kirpichenko 1982, Zhang 2004 and Johansson 2011). Measurements 
in full-scale were mentioned by Kirpichenko (1982). However, no details of the measurement method were given. Newman et al. (2008) measured the final swell by physical means when blasting an ore slice against caved material in a drift at Kiruna mine.

Measurements of the burden dynamics of blasting into a compressible material are sparse. Small-scale tests were carried out by Rustan (1970), Volchenko 1977, Kirpichenko 1982 and more recently by Johansson (2011) and Petropoulos (2011).

\section{BURDEN INSTRUMENTATION OF SLC BLAST RINGS}

Understanding the interaction of confined blasting conditions, blast design and rock mass characteristics on rock breaking performance is rudimentary. Therefore the idea arose to instrument the burden with various sensors to study the effect of confinement on dynamics of burden movement and breakage in full-scale. The conceptual layout for a future test program is shown in Figure 1.

The tests focus on investigating the functioning of the blast in the upper part of the rings. These tests comprise the drilling of boreholes from the longitudinal drifts into the burden area of the blast rings closest to the footwall to study the following:

\section{Dynamics of burden movement}

- Swelling of the blasted burden and compaction of caving debris

- Existence of voids prior to and after blasting

\section{Breakage of burden}

- Verification of over-and underbreak

- Quantification of fragmentation and mobilization

Breakage of burden was examined by static and dynamic Time Domain Reflectometry (TDR) of coaxial cables grouted in boreholes within the burden (Wimmer \& Ouchterlony 2008, Wimmer et al, 2011a). The present paper relates though to the study of burden movement.
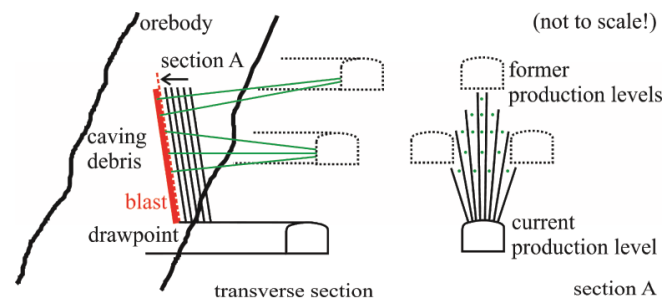

Figure 1. Future burden instrumentation of SLC blast rings.
The implementation of the proposed measurement program in production mining is subject to some complicating factors. The measurement holes have to pass at least $30-40 \mathrm{~m}$ of footwall before they reach the nearest rings. Accurate surveying of the ring holes, careful planning and high-precision drilling are necessary for a successful future field test. Another factor is the correct placement and installation of the measurement systems from the backside of the burden.

Studies of burden movement have been carried out previously with various systems ranging from very simple to advanced ones but with installation in-front of the blast. This study requires measurement of burden movement from the reverse side. All measuring equipment must therefore be installed inside a borehole or alternatively at the borehole collar. In principle, a variety of measurement systems and components available today could probably be used. However, their applicability, reliability and accuracy remain to be assessed for this specific task.

The fact, that the measurement problem is new and the methods used are untested, are the reason that controllable, simplified drift wall blasting experiments were carried out. Main focus was the development of measuring equipment that could be scaled up to future full-scale SLC blasting and installed behind the rings. Due to different conditions these tests to not intend to model full-scale blasts.

\section{DRIFT WALL BLASTING TESTS}

Adjacent pillars in the completed production block 12 on the $691 \mathrm{~m}$ level were chosen for the tests (Wimmer et al. 2011a). Blasting at two sites (test 2, drift 132-130 and 4, drift 127-130) was carried out against artificially filled drifts with a mixture of broken ore and waste loaded by a LHD machine. This way a confined blasting situation should be achieved which also allowed a later inspection of the blast results.

Two blastholes with diameter $115 \mathrm{~mm}$ were drilled for each test parallel with the drift. The tests were extensively instrumented with different sensors which were installed in holes that where drilled from the drift behind into the burden area. Table 1 summarizes the blast design.

The blast sites were well documented in terms of a face survey and structure mapping before and after blasting with the photogrammetry system 'ShapeMetriX3D' (3G Software \& Measurement). All boreholes were surveyed with a gyro based deviation probe 'Flexit SmartTool' (Flexit). As an example, blast site of test 4 is shown in Figure 2. 
Table 1. Blast design, test 2 and 4 with blastholes B2-B5.

\begin{tabular}{lllllll}
\hline & & \multicolumn{2}{c}{ Test 2 } & & \multicolumn{2}{c}{ Test 4 } \\
\cline { 3 - 4 } Parameters & Unit & B2 & B3 & & B4 & B5 \\
\hline Diameter & $\mathrm{mm}$ & 115 & & 115 & \\
Hole length & $\mathrm{m}$ & 13.2 & 11.1 & & 13.0 & 14.0 \\
Charge & $\mathrm{kg} / \mathrm{m}$ & 12.0 & 12.4 & & 11.4 & 11.6 \\
$\quad$ concentration & & & & & \\
Stemming length & $\mathrm{m}$ & 4.0 & 3.0 & & 2.5 & 2.5 \\
Effective burden & $\mathrm{m}$ & 0.9 & 0.8 & & 1.5 & 1.7 \\
Effective spacing & $\mathrm{m}$ & 1.6 & & 2.0 & \\
Delay time & $\mathrm{ms}$ & 0 & & 0 & \\
\hline
\end{tabular}

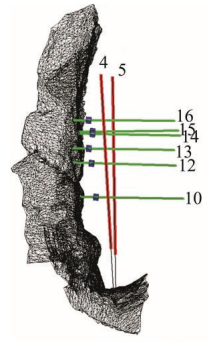

(1)

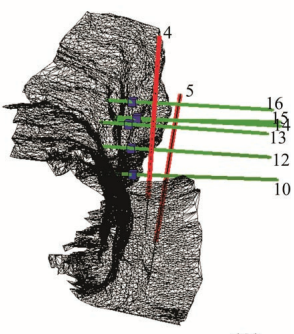

(2)
Figure 2. Measurement points (cubes) within burden for the confined test $4,(1)$ top-view and (2) isometric view.

The boreholes were charged with the emulsion explosive 'KR0500' (Kimulux Repumpable 0500), which is sensitized by glass micro-balloons and contains aluminium. The initiation system consisted of primers 'SLP-50' (Sliding Primer-50) composed of a cap sensitive emulsion packed in a hollow plastic casing. A $25 \mathrm{~g}$ primer made of PETN, wax and oil is placed in the central hole. Programmable electronic detonators ' $\mathrm{i}-\mathrm{kon}^{\mathrm{TM}}$ ' (Orica) were used for initiation. The boreholes were stemmed with sand/gravel.

The Velocity of Detonation (VoD) and vibrations at adjacent pillars were recorded to document detonation of the blastholes. For documentation purposes a camera with high speed filming capacity of $300 \mathrm{fps}$ was used (Casio EX-F1). It helped to verify the functionality of sensor systems installed behind the burden and that the confinement was properly built up. Special attention was given to further document the situation after blasting in terms damages to the sensor systems and to determine final displacement.

\subsection{Measurement systems}

The selection of a proper measurement method to measure the dynamics of burden movement depends upon key factors such as:
- Measurement range of minimum $500 \mathrm{~mm}$ with high sampling frequency

- Simple installation, i.e. inside a borehole or at the borehole collar

- Robustness in a mining environment i.e. shock, vibration, dust, moisture, etc.

- Preferably comprehensive and highly reliable, unbiased results

- Reasonable priced, either single-use or re-usable

Measurement of the confined burden movement in full-scale has been unresolved: the methods used have therefore not previously been tested.

Several types of displacement transducers are described in the technical literature but their suitability to reliably measure burden movement is rather uncertain. Because of this uncertainty and the fact that only a limited number of experiments could be made a redundant instrumentation with various different sensors was made:

\section{Accelerometers}

- Piezoelectric accelerometer ('PE'; Endevco 7255A-01) and piezoresistive accelerometer ('PR'; PCB 35011220KG)

\section{Continuous position sensors}

- Draw wire sensor ('DRAW'; Firstmark Controls 161-2145H-BSS)

- Magnetostrictive position sensor ('MAGNET'; Santest GYcRS with custom-made magnet)

\section{Incremental relative distance sensors}

- Detection of a hole matrix with laser light barrier ('LASER'; Sensor Instruments D-LAS-HS)

- Detection of cable breakage as short circuits ('SPEAR'; Swebrec's 'coaxial cable spear')

- Detection of coding with fibre photoelectric sensor ('FIBRE'; 'fibre-optic zebra gauge')

\subsubsection{Instrumentation}

Test 2 had three boreholes ('M3-M5' with $\varnothing 76 \mathrm{~mm}$ ) and test 4 six holes ('M10-M16' with $\varnothing 76 \mathrm{~mm}$ and $115 \mathrm{~mm}$; Fig. 2) respectively which were instrumented to measure burden movement. In all measurement holes an anchor was gouted mid-way in the burden containing axially mounted 1-axis accelerometers. For signal transmission, steel cables each with three embedded electrical cables have been strain-relieved by a conical termination filled with polyester resin mounted within the anchor. A cable magazine was contained in a casing either behind the burden or at the borehole collar.

\subsubsection{Installation behind the burden}

The incremental relative distance sensors, coaxial cable spear and fibre-optic zebra gauge, could be installed closely behind the burden. Thereby, the 
movement of the anchor was transferred by a threaded hollow pulling tube which also housed the steel cables and was guided in a strong protection casing. A bolting of the latter to a mounting plate at the borehole collar in the neighbouring drift should serve to keep the casing fixed in the holes so that a true relative movement is measured. For the coaxial cable spear the pulling tube had a cutting knife made of hardened steel with an annular gap at the end to gather the cut cable pieces. Coaxial cables were circumferentially mounted within the casing and fed through holes. The spacing of planned cable cuts was successively increased, from fine to coarse resolution at the end of the movement. For the fibre-optic zebra gauge a coded, black-and-white, adhesive foil with strip widths either 5 or $10 \mathrm{~mm}$ was attached to the pulling tube. Movement was detected by 2 fibre photoelectric sensors mounted inside the casing. This would from the sequence of the arriving signals reveal the direction of movement.

\subsubsection{Installation at the borehole collar}

Due to their required space, some of the sensors, i.e. continuous position sensors (draw-wire and magnetostrictive sensor) and the laser-light-barrier were mounted on a stand at the collar.

The movement of the anchor was transferred by the steel cable passing through a flexible teflon coated steel hose which gives both protection and a friction reduction. The sensors were mounted asymmetrically on the stands with a cross-beam to the element housing the cable termination within a guide bar. The cable assembly was pre-tensioned by clamping the cross-beam. The electrical cables from the accelerometers leave the guided element at the rear side to enter a cable magazine from which they could be rapidly extracted. The spring system of the draw-wire sensor is rated for a maximum cable acceleration of $1350 \mathrm{~g}$ which should be sufficient to retract and to tension the cable assembly.

\subsection{Unconfined situation (test 2)}

Filling of the drift for test 2 was not complete, neither in height nor in length, which led to a relatively unconfined blasting situation. This resulted in excessive material movement, upwards and outwards from the drift and left a wide trench in front of the blast site. It was caused by a too conservative assessment of the required filling degree, a small burden and a partly nonexistent opposite drift wall.

The collected data is a result of a combination of factors: the blasting situation, the sensors used and the construction and mounting of the systems. As a consequence the time delays and the characteristics of the curves differ. The velocity and displacement signals are summarized in Figure 3.
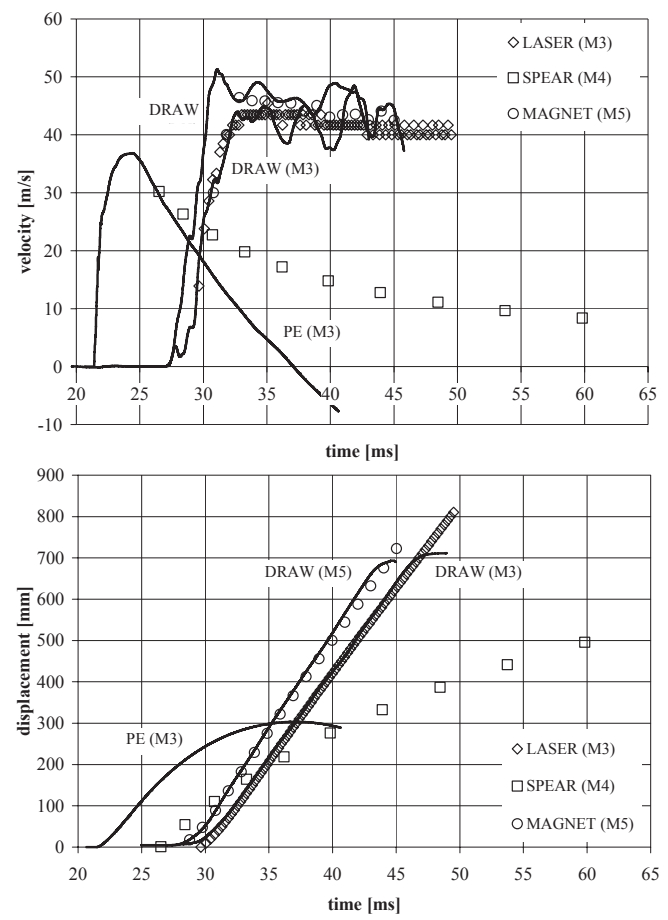

Figure 3. Velocity and displacement, test 2.

With the blastholes detonating almost simultaneously at $20 \mathrm{~ms}$, the anchor bearing the PE accelerometer in hole M3 first moved after $1.9 \mathrm{~ms}$. The instrumentation behind the burden in hole M4 (coaxial cable spear) came $6.5 \mathrm{~ms}$ after detonation and $1 \mathrm{~ms}$ later the instrumentation at the borehole collar of hole M3 and M5 started to move. The comparatively long reaction time for the coaxial cable spear could be based upon its incremental operating mode, i.e. an initial movement is required until the first cable is cut, and a comparatively high weight.

The velocity signals from the PE accelerometer and the coaxial cable spear showed clearly decay in velocity, but the other gauges did not. From the blasting result with massive cast of material an early retardation effect and rigorous physical stop effect can be excluded. Hence an effect of the insufficient filling on the initial measured burden movement and decrease in velocity is doubtful but rather possible error sources for the measurement should be found. There was no linear guiding for the anchor with in-built 1-axis accelerometer. As there was no resistance of masses either most likely the anchor rotated during its trajectory at an early stage so other components increasingly affected the reading. For the coaxial cable spear, it was found that the spear moved unintentionally 
together with a protection casing because concrete penetrated during installation in this casing. The thick-walled casing moved about $1.5 \mathrm{~m}$ from the wall and got stuck as it was considerably bent. It was verified, that the actual measuring tube with installed cables did not move. However, a large frictional effect of the tight-fitting casing within the borehole wall can be assumed which affected the measurement result.

By contrast, the gauges linked to the cable assembly (draw-wire, magnetostrictive sensor and laser-light barrier) did not show any decrease in velocity for the designed measurement range of $700 \mathrm{~mm}$. Good agreement exists between the different measurement methods installed for the same borehole. For hole M3 the mean velocity is around $42 \mathrm{~m} / \mathrm{s}$ and for hole M5 around $45 \mathrm{~m} / \mathrm{s}$. There is a cyclic variation of the velocity for both draw-wire sensors as they reach their maximum level. This might be related to the design of the potentiometer and fast withdrawal of the steel cable. With the blast result in mind it is considered possible that an essential retardation for the initial movement did not exist. According to that it is difficult to assess if the spring system of the draw-wire could on principle counteract the high inertia of the mass.

\subsection{Confined situation (test 4)}

A much better filling was achieved for test 4 and a near confined blasting situation was achieved. A movement on the surface of the muckpile was neither verified during blasting (high-speed filming) nor from post-blast inspections. At about $100 \mathrm{~ms}$ after detonation, some gases started to leak through the pile. Remote inspections through the instrument holes with a long-range borescope and observations during mucking showed that there existed a void between the pillar and the blasted material (Fig. 4).

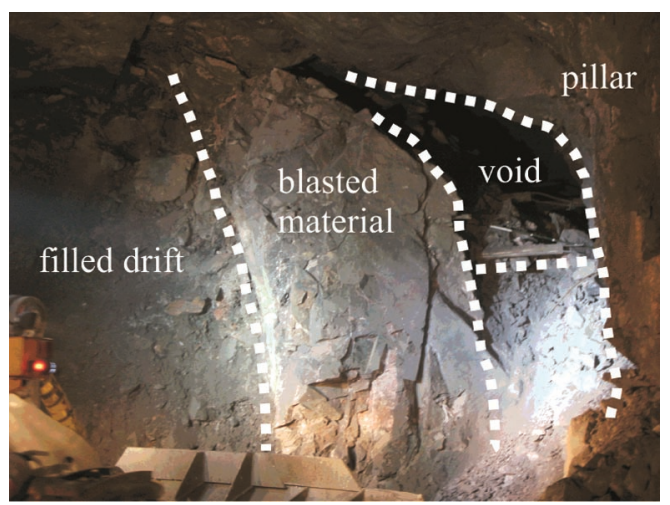

Figure 4. Blast result, test 4.
The blasted material moved about $1.2-1.6 \mathrm{~m}$ in the upper part of the drift section. The compaction is in the order of $4-5 \%$ based on the void in Figure 4. It is likely that the value of compaction could be adjusted upwards as there was partial collapse of the gap before or during the mucking operation. Definitely, the degree of filling and compaction, the simultaneous initiation and the stress situation within the present test differed from the SLC situation. Voids in operational SLC mining were identified to be related to disturbed flow, i.e. 'shallow-draw phenomenon' (Selldén \& Pierce 2004; Power 2004; Wimmer et al, 2012). Nevertheless, it raises the question if a bulking or even voids closest to the ring plane possibly exist also directly after blasting.

During mucking it was also observed that the burden has moved more or less as one unit during blasting. It could be characterized as very coarsely fragmented, and was difficult to muck, with some boulders that were as large as $1.5-2 \mathrm{~m}$.

For test 4, all anchors with built-in accelerometers were linearly guided by a pulling tube within a casing. The post-blast inspection revealed that the anchors were well fastened within the concrete/ borehole and moved with the masses towards the debris. Problematic, however, was that the casings started to move together with the accelerating anchors and the surrounding material. The casing became bent likely at the very beginning due to the shock waves arriving and causing the inner pulling tube to catch. A friction mechanism between casing and the throw of material is also conceivable. The desired relative movement between the inner pulling tube and outer casing, if any, only occurred at the very beginning of the movement. These mechanisms disturbed all systems that were based upon a relative motion between the outer casing, with built-in measurement system, and the pulling tube as the moving part. However, this problem can in the future constructively be solved as solely a buckling-resistant inner pulling tube reaches into the burden and the actual measurement system within the casing is placed well behind the burden.

Reliable measurement results for test 4 were obtained with the accelerometers and draw-wire sensors. The velocity and displacement signals are summarized in Figure 5.

The burden movement during test 4 , as measured with accelerometers, indicated a rapid one- or two-stage acceleration phase to a maximum velocity of $16-32 \mathrm{~m} / \mathrm{s}$, followed by a retardation phase which had a similar character for all gauges. In particular, the curves of the PE accelerometers for the very closely spaced holes M15 and M16 also exhibited very similar curve characteristics for the initial movement. The final stop and its characteristics were not captured as the maximum displacement 

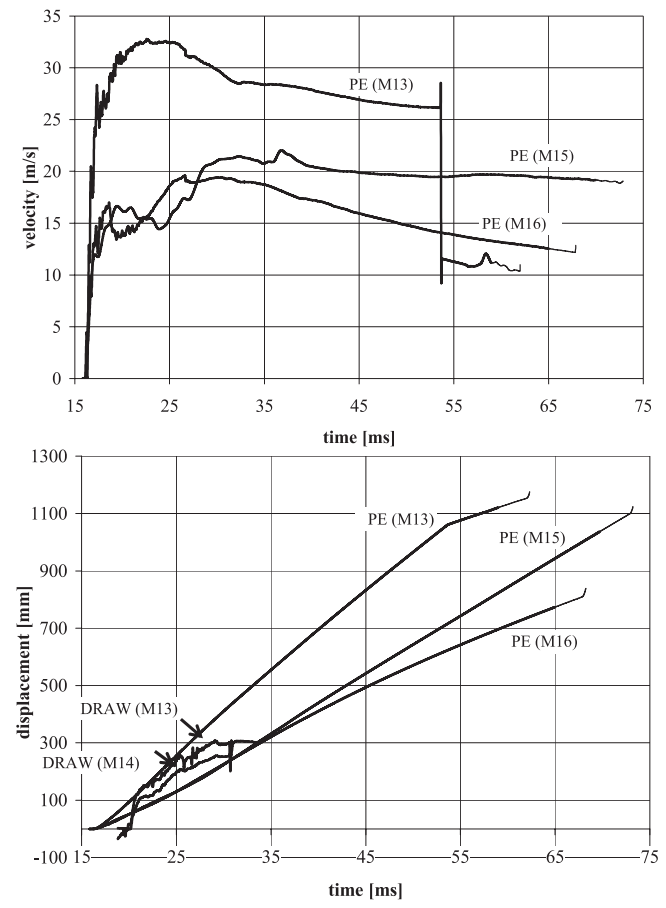

Figure 5. Velocity and displacement, test 4.

of the burden was underestimated. The total displacement measured corresponds to the maximum available cable length of the accelerometer system. It is therefore reasonable to assume that the results obtained up to this point are reliable.

As the entire systems moved violently, i.e. anchor with pulling tube and casing, the bolting to the neighbouring drift did not withstand the forces. Damages occurred to the cabling within the borehole and at the collar to the mounting plates (bent or broken in half). This way, the draw-wire sensors were also badly affected as they did not record more than about $300 \mathrm{~mm}$ of displacement. The draw-wire results did not capture the initial acceleration phase as the signals after a delay of 3-4 ms rise within $0.5-0.6 \mathrm{~ms}$ to the accelerometer levels. The initial jerks and the signal delay of the draw-wire are probably wave propagation phenomena or the associated local acceleration may have exceeded the manufacturer's limits. The displacement records between $20-30 \mathrm{~ms}$ are quite reasonable though compared with the integrated PE accelerometer signals.

\section{$3.4 \quad$ Numerical modeling}

To complement the understanding gained from the burden movement measurements a numerical modeling investigation was also undertaken using the HSBM 'Blo-Up' software. The objectives of the study were twofold: (i) to gain additional insight into the process of blasting in confined conditions and (ii) as a verification study of Blo-Up.

The Hybrid Stress Blasting Model (HSBM) is a high level blast modeling research tool which provides results that can be used implicitly for practical blast design. The code is being developed through an international collaborative research project funded by a consortium of companies which is comprised of explosive, equipment suppliers and major mining companies. A key component of the HSBM is the numerical code and user interface designated as Blo-Up. The Blo-Up software uses a unique combination of continuous and discontinuous numerical methods to represent the key processes occurring in non-ideal detonation, rock fracturing and muckpile formation. The model is described in detail by Furtney et al. 2009.

The flex-boundary condition was developed to model the behaviour at the boundary between the intact burden and the caved material in an SLC ring. This condition exists at the boundary of the model so the caved material is not explicitly represented. The broken rock material in the tests and in real SLC rings are expected to show compaction behaviour in which the modulus increases with displacement and unloading occurs along a stiffer modulus. In order to incorporate this compaction behaviour into the model a non-linear hysteretic spring is parameterized to describe the waste material.

Tests 2 and 4 described herein are analyzed using Blo-Up. Although test 2 was relatively unconfined it provides excellent data on the burden movement. This allows for a calibration of the material properties. The objective is to build confidence in the model by exploring the simpler unconfined case before moving on to the less constrained and more complex case of the confined blasting.

Figure 6 gives a summary of the results of the numerical investigation. In both the unconfined conditions of test 2 and the confined conditions of test 4 , Blo- $U p$ reproduces the burden velocity and displacement observed in the experiment. In the unconfined case the burden accelerates to a velocity of about $45 \mathrm{~m} / \mathrm{s}$ over a period of approximately $1 \mathrm{~ms}$. In the confined case, the maximum velocity reached at the same point in the burden is about $30 \mathrm{~m} / \mathrm{s}$. The expansion energy of the explosive product gasses is consumed in overcoming interlocking in the burden and in accelerating the burden mass. In the confined case, the expansion energy is also consumed in compacting the caved 

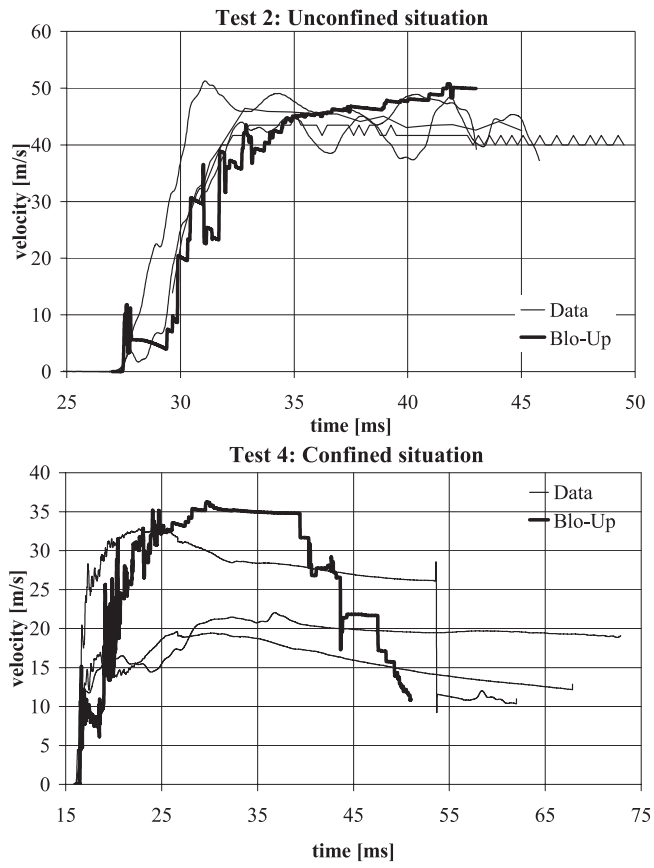

Figure 6. Comparison of burden velocity measurements and Blo-Up model predictions.

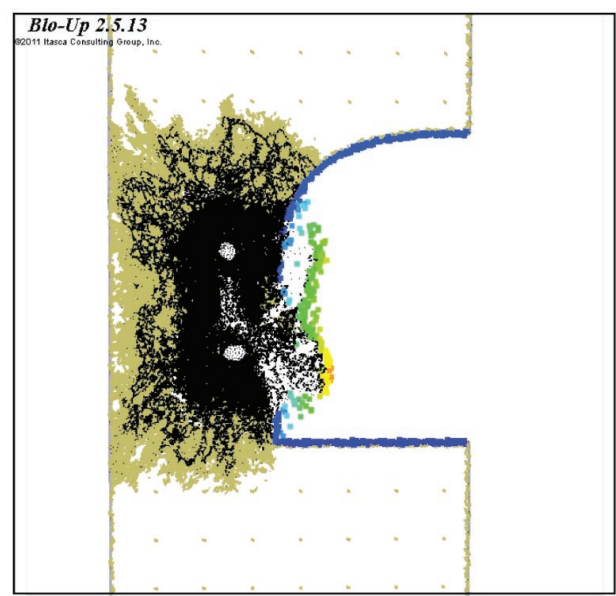

Figure 7. Model predictions of burden swell. Contours are for displacements of 0 to $1.2 \mathrm{~m}, 60 \mathrm{~ms}$ after detonation.

material, resulting in smaller burden velocities and displacements.

Figure 7 is a cross section of the confined model showing the boundary swell, fracturing and back break. As in the field measurements a gap centered on the boreholes is observed.

\section{DEVELOPMENT OF A MEASUREMENT SYSTEM FOR BURDEN MOVEMENT}

Based upon the experiences of the drift wall blasting blasts and parallel on-going laboratory tests, an incremental relative measurement system installed closely behind the burden was put into use. The main idea is to have a system which captures both the fast acceleration phase and the slower movement until rest. The measurement system consists of a hollow pulling tube with an anchor incorporating an accelerometer suited for long duration transients, which is grouted inside the burden. Behind the burden, a displacement measuring system with fibre-optic sensors is mounted in a shock-absorbent casing, which is kept in position. A coded tape is fastened to the pulling tube and detected by two fibre photoelectric sensors. This allows detection of a change in the direction of movement. Figure 8 illustrates the essential parts of the measurement system as applied in the last field test.

\subsection{Accelerometers}

Both, piezoelectric (PE) and piezoresistive (PR) accelerometers are widely used shock accelerometers. However, their applicability for integration of the signal to velocity and displacement is often troublesome and related to specific construction-conditioned problems (Chu 1992).

Despite that PR accelerometers were improved considerably in their usable bandwidth $(>500 \mathrm{kHz})$ and rigidity, the sensor can still be set into resonance as the gauge mechanism is practically undamped. The result of this failure is complete loss of data and eventually permanent gauge damage. On the other side, $\mathrm{PE}$ accelerometers are more robust under the same conditions but have other deficiencies:

- Inability to hold their charge output during a steady input (DC response).

- 'Zero-shift', i.e. failure of a sensor's output returning to zero after a dynamic event.

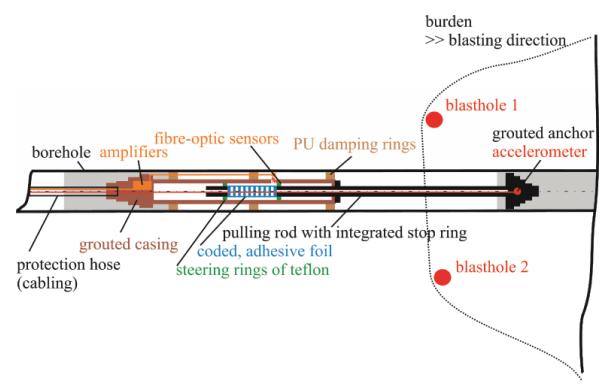

Figure 8. Measurement system installed behind the burden. 
The phenomenon of zero-shift arises during an explosively generated shock (pyroshock), as a unidirectional shift of unpredictable polarity and amplitude. Measures to reduce the effects of zeroshift are the design of the accelerometer, i.e. avoidance of overstress of sensing element and sensor part movements as well as care in the installation and set-up, i.e. avoidance of frictionally generated cable noise (triboelectric effect) and base strain.

For example, the design of the PE accelerometer, 7255A-01 (Endevco) is an attempt to eliminate the zero-shift problem. It incorporates a built-in mechanical filter for sensor isolation and a matched electronic low-pass filter in order to remove unwanted residual ringing superimposed on the accelerometer output signals. Several independent test results have shown that the design virtually eliminated the difficulty of zero-shift (Chu 1988, Yiannakopoulos \& Van der Schaaf 1998).

\subsection{Fibre-optic zebra gange}

The usage of incremental relative distance sensors to determine the time for initial face movement and the rate of burden movement is not novel within the field of blasting. Different systems which yield time-distance data as detection of cable breakage were successfully applied by Noren (1956) and Olsson et al. (2009).

The fibre-optic zebra gauge might be regarded as a direct improvement. A fibre photoelectric sensor detects the movement of the object by the detection of a coding printed on adhesive foil. Detection occurs when the light beam, incident on the target, is reflected by the target and received. The optical plastic-fibre consists of a transmitter (core, $\varnothing 1 \mathrm{~mm}$ ) surrounded by 16 tiny $(\varnothing 0.26 \mathrm{~mm})$ receiver fibres embedded in a flexible end tip (Sick LL3-DB02).

\subsection{Laboratory tests}

Laboratory tests with the aim to evaluate the performance of different accelerometers and compare their integration results with incremental relative displacement sensors (coaxial cable spear, fibreoptic system) were carried out.

The test set-up was intentionally kept simple and robust with regard to its latter application in the mining environment. A probe that housed the accelerometer was guided within transparent PVC tubing $(\varnothing 40 \mathrm{~mm})$ mounted in a lathe.

The probe was pushed forward by a tube guiding the cables and inserted through the hollow work spindle. In this way a constant velocity in the range of $3-6 \mathrm{~m} / \mathrm{s}$ could be achieved for a certain distance. The measurement range was varied between 150 and $750 \mathrm{~mm}$ and the characteristics of stoppage could be differently simulated. The integrated accelerometer signals were compared to velocity and displacement measured at the probe by the incremental relative distance sensors.

The incipient use of the cable breakage method proved to be difficult as the cutting of coaxial cables induced ripples in the acceleration signal. Consequently, a comparison with conventionally measured velocity and displacement was largely incoherent.

By contrast, the non contact fibre-optic zebra gauge did not exert any influence on the acceleration signal. It also allowed detection of the direction of movement with two systems mounted in a sequence and gave an increased resolution to identify the acceleration and retardation phases.

The results from the PE unit compared with the PR unit looked very much alike, see Figure 9.

The integrated data coincided well with the velocity and displacement as measured by the fibre optics. The actual stop was recognizable as a transient spike in the velocity and a kink in displacement just before $2.10 \mathrm{~s}$. For the time window of interest $(50-150 \mathrm{~ms})$, zero-shift did not prove significant. Continuing though to integrate the signal of the PE unit after the stop revealed later problems of zero-shift. This appeared as a ramp in velocity and a parabola in displacement. This problem was
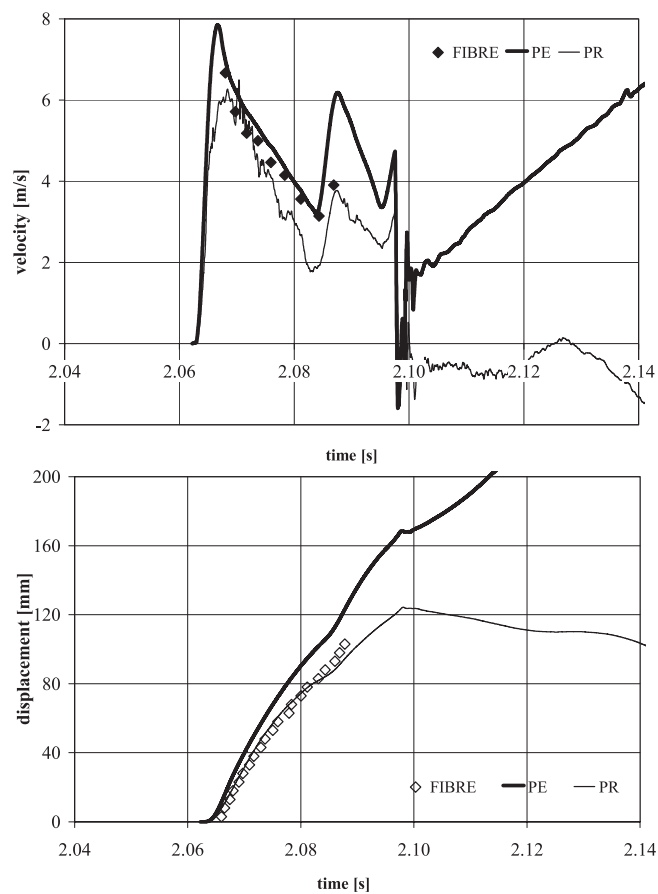

Figure 9. Velocity and displacement, PE and PR accelerometer compared with fibre-optic gauge, abrupt stop at $150 \mathrm{~mm}$. 
also directly noticeable when comparing the mean offset before (1) to after the shock event (2). The mean $2 / 1$ is 0.72 for the $\mathrm{PE}$ and 1.01 respectively for the PR unit (18 measurements). Even so, the PR unit did not perform better after shock.

The dynamics from a burden movement against confinement in terms of rock masses would presumably look different with a) a much higher acceleration and initial velocity and b) a gradual retardation effect as the confining masses get compacted. These may also react as a spring system. For the latter reason the used stop in the form of hard plastic was replaced by a compression spring. A pronounced bounce effect and damping which reached a peak indicating the stoppage of the probe could be found, see Figure 10. Further experiencing with stopping systems of different rigidity indicated that a harder stop was easier to identify as a clear stop.

The use of PR accelerometers required some special care. Attention was paid to the cable selection in terms of special treatment to reduce triboelectric noise. In particular, for the PR unit it was essential to use low-pass filtering $(10 \mathrm{kHz})$.

By contrast, the PE accelerometer type is reliable due to a secure mounting arrangement, ease of use, robustness and noise free, consistent results in the laboratory tests. The excellent performance of the PE unit is due to an effectively working in-built mechanical filter in combination with an electronic low-pass filter. Comparing displacements and velocities derived by the PE unit with the fibre-optic measurements pointed to a reasonable agreement. On average, the velocity was increased by $7 \pm 37 \%$ (165 meas.) and the displacement decreased by $9 \pm 11 \%$ (378 meas.) as the actual values.

\subsection{Field tests}

For further development of the described fibreoptic system, collaboration with the project 'Rock support subjected to dynamic loading'

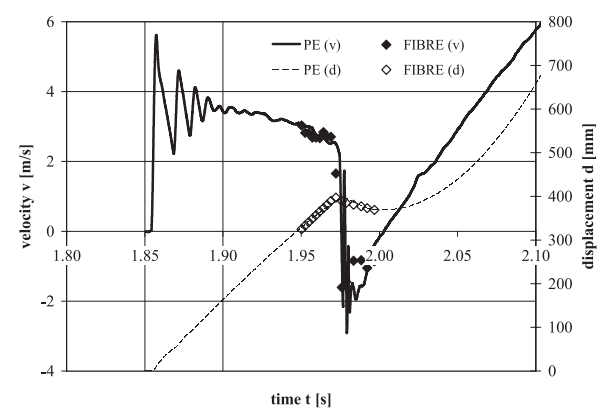

Figure 10. Velocity (v) and displacement (d), PE accelerometer compared with fibre-optic gauge, suspended stop at $400 \mathrm{~mm}$.
(Shirzadegan 2011) was valuable. In the test series the effectiveness of various ground support systems under dynamic loading conditions are tested. Rock burst loading is simulated using explosives in holes drilled behind differently reinforced drift walls. The reinforcement was of integrated character, i.e. rock bolts, shotcrete and wire mesh. The equivalence of rock bursts and blast-generated dynamic load is doubtful. However, the tests with an artificially generated initial movement and a possible rebound effect were ideal for the development of a reliable technique to measure face velocity and displacement in a comparatively simple and controllable way. Two tests were carried out at block 9, on the $741 \mathrm{~m}$ level at the Kiruna mine (Wimmer et al, 2011b). Both were single-hole shots with instrumentation either in front of the burden (test 5, drift 93, right side), or in front and behind the burden (test 6, drift 95-98).

In test 5 , a movement of at most about $0.1 \mathrm{~m}$ with a subsequent gentle rebound was recorded. In test 6 , the limit case of breakage was achieved as the burden for the section of lower charge concentration moved slowly as a single block for about $0.5-1 \mathrm{~m}$. The section with higher charge was unexpectedly broken but showed rather coarse fragmentation.

The fibre-optic zebra gauge worked reliably and its output was comparable with other measuring techniques when installed in front of the burden. Velocity and displacement derived from it were compared to a laser distance sensor (Fig. 11).

The field tests showed also that the tested accelerometers were not suited as a stand-alone technique to yield information on the complete burden movement but are rather complementary. A splicing of the initial accelerometer data with the results of the fibre-optic gauge might be the best option.

Figure 12 compares velocity and displacement from the integrated accelerometer signal with the

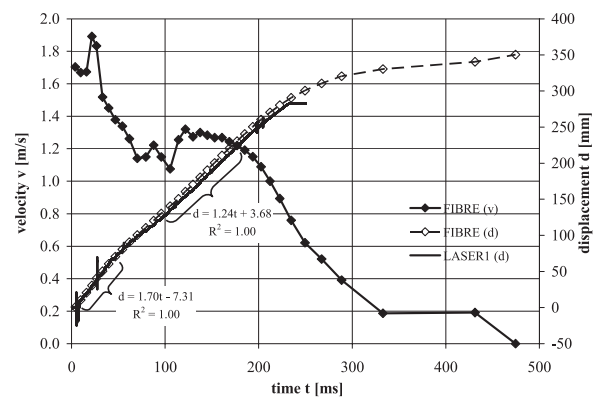

Figure 11. Test 6, velocity (v) and displacement (d), laser distance sensor (Sick OD2-250W150) and fibreoptic gauge. 


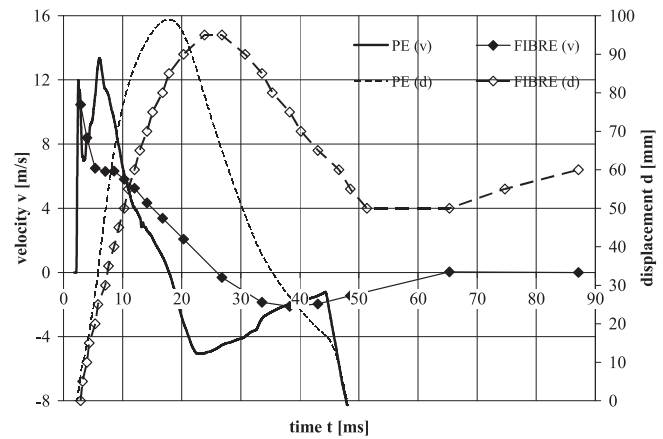

Figure 12. Test 5, velocity (v) and displacement (d), $\mathrm{PE}$ accelerometer and fibre-optic gauge.

fibre-optic gauge. The PE accelerometer picked up the initial wall velocity as well as its maximum displacement and gives a result comparable to that of the fibre-optic gauge. The observed double peak in velocity for the wall mounted accelerometer might have a physical explanation. It is reasonable to assume that this represents the arrival of shock wave at the drift face followed by initial fracture propagation and/or a reflection from the boundaries of the pillar. A similar observation was made for a draw-wire sensor mounted at the same section of the wall but further down to the floor.

Possible explanations for the later discrepancy between the integration result of the accelerometer signal and the fibre-optic gauge are i) a zero-shift problem occurring at a very early stage, ii) mounting issues for the fibre-optic gauge, i.e. a lifting of the floor and subsequent low-frequency oscillation of the mounting stand which was also observed from high-speed filming (at $\sim 25-35 \mathrm{~ms}$ ).

The excellent performance of the PE accelerometers achieved in the laboratory tests could not be repeated for the field tests. It was observed that their failure is often related to specific spurious pulses in the signal that occur during the movement and mask the actual signal in the low frequency region. The expected difference in behaviour of the PE and the PR accelerometers in the field did not materialise. If such an effect exists it may be apparent during very slow motion and as motion ceases.

With the measurement system installed behind the burden, the initial face velocity was measured. Then, as a result of the blasthole located nearby and penetrating gases, a blow-out of the system occurred. The blow-out forced the fibre-sensing backwards and accelerated the apparent outward movement of the anchor. A curve splice of the initial integrated accelerometer signal and the later fibre-optic signal would yield the whole time history of the movement, see Figure 13. As the

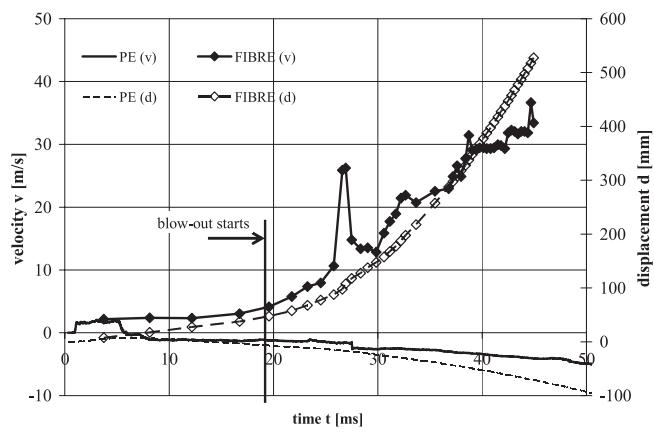

Figure 13. Test 6, velocity (v) and displacement (d), $\mathrm{PE}$ accelerometer and fibre-optic gauge.

fibre-optic gauge measured a relative movement between the anchor and the casing, two relevant mounting matters need to be solved in the future. The starting position needs to be well defined and casing movement during measurement prevented. Important constructive measures are a grouted casing as well as an integrated stop ring on the pulling tube (Fig. 8).

\section{CONCLUDING REMARKS}

The instrumentation of the burden to study the dynamic process of blasting into a compressible material is a crucial factor to quantify the blast function within a SLC ring. This development work was initiated so that SLC blasting may in the long term be better understood. Different redundant sensor systems were tested in both unconfined and confined drift wall blasting tests. Blo-Up accurately reproduced the burden velocity and displacement observed in the experiments. The measurement methods deployed in the tests were further developed and tested. The novel system, a $\mathrm{PE}$ accelerometer combined with the fibre-optic zebra gauge, will be further used to measure confined burden movement in filled drift wall blasting tests under controlled situations and a full-scale SLC instrumentation is planned. Moreover, the Blo-Up software might be used in the near future to gain additional insight into the complex process of blasting SLC rings.

\section{ACKNOWLEDGEMENTS}

The Hjalmar Lundbohm Research Centre (HLRC), a research foundation at LTU and endowed by LKAB is thanked for its financial support throughout the $\mathrm{PhD}$ project 'Improved break- 
age and flow in sublevel caving'. The colleagues from the central workshop and Complab at LTU are thanked for the support and numerous valuable discussions related to the development of the measurement equipment. Special thanks go to our technicians and the mine surveyors in the LKAB Kiruna mine for all their help consistently provided in practical matters.

\section{REFERENCES}

Belen'kii, E.V., Kovtun, I.N. \& Fedorenko, P.I. 1969. Buffer properties of caved ore. Sov Min Sci, 5(4): $444-447$.

Brunton, I.A. 2009. The impact of blasting on sublevel caving flow behaviour and recovery (Doctoral thesis). Univ of Queensland, Brisbane.

Chu, A.S. 1988. Built-in mechanical filter in a shock accelerometer. In 59th Shock and Vibration Symp (1: 251-269). Washington, USA: Shock and Vibration Information Centre, U.S. Naval Research Centre.

Chu, A.S. 1992. Problems in high-shock measurement (Paper TP 308). San Juan Capistrano: Endevco Corporation.

Cullum, A.J. 1974. The effects of confined blasting on rock fragmentation and flow characteristics in sublevel caving (Master thesis). Univ of Queensland, Brisbane.

Furtney, J., Cundall, P.A. \& Chitombo, G. 2009. Developments in numerical modeling of blast induced rock fragmentation: Updates from the HSBM project. In J.A. Sanchidrián (ed.), 9th Int Symp on Rock Fragmentation by Blasting (335-342). London: CRC Press.

Johansson, D. 2011. Effects of confinement and initiation delay on fragmentation and waste rock compaction. (Doctoral thesis). Luleå Univ of Technology, Luleå.

Kirpichenko, V.M. 1982. Study of ore breaking in a compressed medium by sets of parallel adjacent bores in the Tash Tagol Mine. Sov Min Sci, 18(3): 233-237.

Newman, T., Hustrulid, W. \& Quinteiro C. 2008. Sublevel caving trial-monitoring effects from blasting an ore slice against caved rock at LKAB's Kiruna mine, Sweden. In H. Schunnesson \& E. Nordlund (eds.), 5th Int Conf and Exhib on Mass Mining (705-713). Luleå: Univ of Technology.

Noren, C.H. 1956. Blasting experiments in granite rock. Colorado School of Mines Quarterly, 51(3): 213-225.

Olsson, M., Nyberg, U. \& Fjellborg, S. 2009. Controlled fragmentation in sublevel caving-first tests (Swebrec Report 2009:2). Luleå, Sweden: Luleå Univ of Technology. In Swedish.

Petropoulos, N. 2011. Influence of confinement on fragmentation and investigation of the burden movement-small scale tests. (Master thesis). Luleå Univ of Technology, Luleå.

Power, G. 2004. Modeling granular flow in caving mines: large scale physical modeling and full scale experiments. (Doctoral thesis). Univ of Queensland, Brisbane.
Rustan, A. 1970. Theoretical basics of the volume-weightmethod for the determination of the ore content of a mixture of blasted ore and waste rock. Kinematics, swelling, loosening and fragment size in the burden for confined blasting in model-scale. (Licentiate thesis). Royal Institute of Technology, Stockholm. In Swedish.

Selldén, H. \& Pierce, M. 2004. PFC3D modeling of flow behaviour in sublevel caving. In A. Karzulovic \& M.A. Alafaro (eds.), 4th Int Conf and Exhib on Mass Mining (201-214). Santiago: Instituto de Ingenieros de Chile.

Shirzadegan, S., Nordlund, E., Nyberg, U., Zhang, P. \& Malmgren, L. 2011: Rock support subjected to dynamic loading: Field testing of ground support using simulated rock burst. In Q. Quian \& Y Zhou (eds.), Harmonising rock engineering and the environment, 12th International Society for Rock Mechanics International Congress on Rock Mechanics (12691273), Leiden, Netherlands: CRC Press/Balkema.

Volchenko, N.G. 1977. Influence of charge arrangement geometry and short-delay blasting on the crushing indices in compression blasting. Sov Min Sci, 13(5): 488-493.

Wimmer, M. \& Ouchterlony, F. 2008. Application of time domain reflectometry (TDR) for block- and sublevel caving mines - State-of-the-art and preliminary laboratory shear tests (Swebrec Report 2008:P3). Luleå, Sweden: Luleå Univ of Technology.

Wimmer, M. \& Ouchterlony, F. 2011a. Study of burden movement in confined drift wall blasting tests in block 12, 691 m level, Kiruna mine (Swebrec Report 2011:P1). Luleå, Sweden: Luleå Univ of Technology.

Wimmer, M. \& Nordqvist, A., Ouchterlony, F. 2011 b. Burden movement while blasting under constraintsTests with new gauges in block 9, $741 \mathrm{~m}$ level, Kiruna mine (Swebrec Report 2011:P2). Luleå, Sweden: Luleå Univ of Technology.

Wimmer, M., Nordqvist, A., Ouchterlony, F., Selldén, H. \& Lenz, G. 2012. 3D mapping of sublevel caving (SLC) blast rings and ore flow disturbances in the LKAB Kiruna mine. In G. Baiden \& Y. Bissiri (eds.), 6th Int Conf and Exhib on Mass Mining (CD). Sudbury: Laurentian Univ.

Yiannakopoulos, G. \& van der Schaaf, P.J. 1998. Evaluation of accelerometer mechanical filters on submerged cylinders near an underwater explosion. Shock and Vibration, 5(4): 255-265.

Zhang, G. 2004. Behaviour of caved ore mass in sublevel caving and its effect on ore dilution. In A. Karzulovic \& M.A. Alafaro (eds.), 4th Int Conf and Exhib on Mass Mining (238-242). Santiago, Chile: Instituto de Ingenieros de Chile. 